\title{
Factors influencing intestinal uptake of food antigens
}

\author{
By Roland J. Levinsky, Institute of Child Health, 30 Guilford Street, London \\ $W C \mathbf{I}$
}

The main function of the gastrointestinal tract is to provide a route for the active absorption of digested nutrients. A second important function is to maintain a barrier between the gastrointestinal contents of the body tissues since the intestinal tract is continually exposed to a variety of ingested antigens not all of which are rendered harmless by the digestive processes. The first line of defence against antigen penetration rests on the integrity of this physical barrier but, in addition, a sophisticated system of non-immune factors, non-specific immune defences and specific humoral and cellular immune responses have developed to cope with the antigenic onslaught. This barrier is incomplete for there is now ample evidence that food proteins may be absorbed antigenically intact into the circulation in healthy people. Although small, the amounts absorbed are sufficient to immunize, since antibodies to food proteins may be demonstrated in most people. Despite the presence of the circulating antibodies, no adverse reactions occur in the majority of individuals in response to antigen entry. Oral immunization elicits a local mucosal immune response which substantially reduces antigen entry; it also results in the state of systemic tolerance in which the individual is incapable of mounting an appropriate antibody response when the same antigen is subsequently given parenterally. It is only when there is a breakdown of these protective homeostatic mechanisms that hypersensitivity reactions to food proteins occur (reviewed by Bienenstock, 1982; Walker, 1982).

\section{Factors preventing intestinal food antigen uptake}

I. Non-specific mechanisms. The non-immunological factors, such as lysozyme ( $E C$ 3.2.1.17), peroxidase ( $E C$ I.1 I.1.7) and lactoferrin in saliva, as well as the acidity within the stomach, serve mainly to protect against colonization of micro-organisms. Similarly, glycoproteins in mucous which coats microorganisms, thereby inhibiting their attachment to the epithelial surface and normal peristaltic mechanisms, prevent the overgrowth of intestinal pathogens. The importance of a normal intestinal microflora cannot be over-emphasized; achlorhidria is associated with an increased proliferation of gram-positive organisms in the small intestine and also with hypersensitivity reactions.

In addition, there are large numbers of cells such as polymorphs in saliva, intra-epithelial lymphocytes in the mucosa of the small intestine, macrophages in the domes and follicles of Peyer's patches and mast cells in the lamina propria of the intestine which function in a non-specific way to inhibit proliferation of 
micro-organisms within the gut. These non-specific defences work synergistically with the more specific immunological defence mechanisms of the mucosa-associated lymphoid tissue (MALT).

2. Specific immune mechanisms: MALT. Two types of lymphoid aggregate are found in the intestine. Multiple aggregates known as Peyer's patches are found in the small intestine, whereas solitary lymphoid nodules are scattered throughout the small and large intestine. Both these aggregates possess specialized epithelial cells (M cell) which are thought to facilitate transport of antigen to the intestinal lymphoid tissue (Owen \& Jones, 1974). The cells of the mucosa-associated lymphoid system, once triggered by antigens, re-circulate and, although localizing predominantly within the tissues from which they originate, also localize in other mucosa-associated areas, such as the mammary gland, the bronchus, the salivary gland and the cervix. Hence lactating human females given non-pathogenic Esherichia coli produce specific IgA antibody-secreting cells in their breast milk. Such antigen priming in the gastrointestinal tract and subsequent seeding to the so-called sterile areas of MALT may provide the mechanism by which the cells maintain special immunity function in these distant sites. The $T$ cells in the Peyer's patches and the mesenteric lymph nodes have a function peculiar to the mucosa-associated lymphoid system. These cells facilitate the production of IgA-immunoglobulin-class antibody, while at the same time supressing IgG- and IgM-immunoglobulin classes (Richman et al. $198 \mathrm{I}$ ).

Antibodies in secretions. Secretory IgA represents the predominant antibody class in intestinal secretions. It consists of two molecules of monomeric IgA joined by a covalently-linked peptide named J chain. The dimeric molecule formed by the plasma cells in the lamina propria is transferred to the epithelial cells where it combines with a transport protein (secretory piece) found in the lateral and basal membranes of the epithelial cells. Secretory piece transports the molecule across the cell and secretory $\operatorname{Ig} A$ is secreted into the lumen by reverse pinocytosis where it acts, together with mucous, as a 'mucosal paint' resistant to proteolytic digestion. Antigen, when trapped at the mucosal surface as an antigen-secretory IgA complex, is rendered more susceptible to degradation by pancreatic enzymes. Furthermore, such complexes may cause goblet cells to secrete mucous which additionally prevents antigen entry (Walker, 1982 ). IgA as a class of antibody does not fix complement by the classical or alternative pathway; its major mechanism of action may be through its capacity to inhibit the adherence of bacteria and viruses to epithelial surfaces, thereby preventing colonization, but it is also capable of immune exclusion of food-protein antigens. Serum IgA, whether in polymeric or monomeric form, may be capable of immune elimination of enterically-derived antigens, and the lack of damaging reactions provided by IgA antigen-antibody complexes may provide a safe route for the small amount of macromolecular antigen which enters the circulation intact.

3. Gut closure. In many animal species the neonatal gut is freely permeable to macromolecules. This is probably necessary because passive immunity from the mother is acquired from breast milk in varying amounts in different species. In 
cows there is free absorption of all macromolecular proteins for the first few days of life, after which the mucosal cells come together to form tight junctions, a process known as gut closure which is enhanced by certain factors in colostrum (Walker, 1982). In rodents there is an active selective transport of immunoglobulins across the mucosal cell for the first 3 weeks of life, and gut closure occurs just before weaning. The human neonate acquires its passive IgG transplacentally in the last trimester of pregnancy and therefore there is no selective advantage in a gut closure mechanism. Studies of milk antigens crossing the gastrointestinal tract in the premature human infant indicate that much greater amounts cross the gastrointestinal tract into the circulation in the very premature, but at full gestational age the amounts absorbed are no greater than those found in older children and adults given equivalent antigenic load in proportion to their body-weight. In the human neonate, breast-feeding does not appear to enhance the relatively-slow maturation which occurs before birth (Roberton et al. 1982). Once gut closure has occurred, the amount of antigens crossing the intestinal mucosa is substantially reduced by the local non-specific immune mechanisms. The immune exclusion provided by secretory IgA is not total and some macromolecular absorption still occurs in all healthy subjects. There may also be a genetic variability in the immune exclusion mechanisms since strain differences have been reported in mice for both immune exclusion at mucosal surfaces as well as for antigen elimination from the circulation.

\section{Mechanisms of transport}

The specialized $M$ cells overlying the Peyer's patches transport antigens for presentation to the mucosa-associated lymphoid system, but antigen also enters the portal and lymphatic circulation by transport across the enterocyte. This process is an active one since it can be blocked by metabolic inhibitors. Protein is endocytosed at the luminal surface of the cell to form a phagosome which coalesces with lysosomes. Some antigen degradation occurs within these but the remainder of the macromolecular protein enters the lateral spaces between the mucosal cells by the process of exocytosis and from there is transported to the portal and lymphatic circulations (Walker, 1982 ). The amount absorbed is in the order of I: 10000 to I:50000 of the antigen load given orally.

\section{Immune elimination and oral tolerance}

The state of systemic hyporesponsiveness to an antigen initially presented orally appears to be the physiological way of preventing harmful reactions occurring due to food proteins. Healthy people have serum antibodies to food proteins but only to low titres and they are not of the variety which elicit hypersensitivity reactions. The mechanisms underlying this orally-induced tolerance are unclear and there may be differences according to the different types of antigens. In mice it has been shown that serum factors are involved since tolerance may be transferred passively to mice that have not previously been given the antigen. One such serum factor is IgG $\mathrm{I}$-subclass immunoglobulin (Chalon et al. 1979). Circulating suppressor $\mathrm{T}$ cells 
may be generated by oral antigen administration in animals but this is only transient. Antigen-specific T suppressor cells are produced in Peyer's patches, then in mesenteric lymph nodes and the spleen. However, within MALT only IgG and IgM production is suppressed, whereas IgA production is enhanced (Richman et al. 1981). Peyer's patches and tonsillar lymphocytes respond to enteric antigens with preferential IgA-class antibodies (Paganelli \& Levinsky, I98I), hence it is likely that the route of antigen entry as well as the unique micro-environment of the mucosa-associated lymphoid system are important for the continued production of IgA antibodies. Interestingly, antigen processing by gut cells may be necessary for the generation of the suppressor cells since this can be transferred in mice by serum from mice rendered orally tolerant, but abrogated by cyclophosphamide pretreatment of the recipient animals (Strobel et al. 1983). $_{3}$ ).

It has also been suggested that prostaglandin synthesis by macrophages may be involved in the regulation process leading to oral tolerance. These highly complicated homeostatic mechanisms are probably maintained by an interaction of serum and cellular factors. Serum IgA may also be effective in immune elimination in the portal and systemic circulation. In normal healthy individuals it has been shown that after drinking milk the antigen entering the circulation is incorporated into IgA immune complexes (Paganelli et al. 1983). In rats and other animals an active transport system for dimeric IgA from blood by secretory piece on the sinusoidal aspect of the hepatocyte to bile has been demonstrated. The animal studies indicate that the liver is an important organ for the clearance of IgA immune complexes formed by antigen absorption from the gastrointestinal tract (Russell et al. 1981). Although this has not yet been shown for humans, the evidence suggests that serum IgA antibodies may participate in clearing such antigens from the circulation by the formation of IgA antibody-antigen complexes and subsequent transport into bile. This non-damaging form of immune elimination would form part of the tolerance process.

\section{Relevance of food antigen entry to disease}

Food allergy may be considered as a major breakdown of oral food tolerance. The symptoms of gastrointestinal allergy may either be local within the gastrointestinal tract, i.e. diarrhoea, bleeding or protein-losing enteropathy, such as occurs in cow's-milk allergy; or systemic, ranging from anaphylaxis, urticaria, asthma, eczema and migraine. The factors underlying the development of gastrointestinal allergy are unclear but there is certainly a strong association with immunodeficiency. Of the specific humoral or cellular immunodeficiencies, selective IgA deficiency affecting about one in 500 of the population, is the most common. Most affected individuals are not symptomatic and the relative lack of gastrointestinal problems could possibly be due to the compensatory role played by increased secretory IgM production. However, these subjects have a high incidence of food allergies and increased food antigen absorption (Cunningham-Rundles et al. 1978). It is unlikely that the development of food allergy is due to excessive stimulation of the immune system by large amounts of antigen crossing the 
gastrointestinal mucosa. The very large amounts absorbed by premature infants is against this; the genetic make-up of the individual is of paramount importance but, in addition, it is likely that the timing of antigen exposure and whether the antigen is presented together with an adjuvant are critical. In the rat, IgE responses may only be elicited by the oral route if a small dose of antigen is presented together with bacterial lipopolysaccharide (Jarrett et al. 1976). In the bottle-fed neonate, bacterial colonization of the gut with $E$. coli could provide the necessary adjuvant, but sensitization would only occur in the vulnerable child. Transient or minor immunodeficiencies may predispose to susceptibility, and a gastrointestinal infection at a critical time may be sufficient to present the antigen in such a form that a hypersensitivity state occurs. The frequent association of gastrointestinal allergy following a gastrointestinal infection, particularly in the neonatal period, may be related to sensitization occurring at that time. The fact that these infants often continue to develop further sensitivities to other food substances may be explained by the observations in allergic rats, where intestinal anaphylaxis facilitates increased uptake of non-specific antigens and subsequent hypersensitivity reactions to them (Bloch \& Walker, 1981). Whatever the mechanism underlying gastrointestinal allergy, following the ingestion of the food substance to which the individual is sensitive there is excessive entry of these proteins into the circulation. In normal healthy individuals such food proteins are cleared by the formation of IgA immune complexes but in the allergic individual a potentially-damaging variety of IgG-IgE and complement-binding immune complex develops after such antigen challenge (Paganelli et al. 1983). The appearances of these complexes have been shown to correlate with the development of symptoms such as itching and wheezing. It has been suggested that triggering of IgE-sensitized mast cells in the mucosa and lamina propria of the gut renders the mucosal lining more permeable to food antigens, since the mast cells degranulate to release vaso-active amines, thereby increasing local vascular permeability. Prior treatment with oral sodium cromoglycate, a drug which stabilizes mast-cell membranes and prevents degranulation, abolishes the symptoms, substantially reduces antigen entry and prevents the formation of these potentially damaging immune complexes. Hence the mechanisms of damage in food allergy may be due to both a type I (immediate) and type III (immune complex) hypersensitivity reaction.

Atopic eczema is thought to be due to food allergy, and in children antigens contained in milk or eggs, or both, frequently provoke symptoms. However, there is increasing awareness that many other diseases may be provoked or exacerbated by food allergy. Controlled double-blind challenges of food substances in children with migraine have shown the strong association of a food allergy in these patients (Egger et al. 1984). There are also anecdotal reports of sensitivity to food substances causing psychiatric disturbances with the mood and behaviour changes being abolished by the avoidance of the offending food (Tryphonas \& Trites, 1979). Similarly, there are reports of patients with rheumatoid arthritis in whom joint symptoms are substantially improved by diets free of certain foods. The 
relevance of food antigen absorption in gastrointestinal diseases, such as coeliac disease, inflamatory bowel diseases, and even children with malnutrition (all conditions associated with a high incidence of precipitating antifood antibodies) is as yet far from clear. The critical question to answer in food allergy is what causes a breakdown in oral food tolerance and what can be done to restore the balance? That there is an alteration in immune responsiveness is clear but apart from avoidance of the offending antigen, there is little that can be done in the way of ireatment as yet. Trials of drugs which regulate the immune system such as thymic-hormone preparations have not been of any benefit for the treatment of conditions such as atopic eczema. It is hoped that in time, with more understanding of the factors regulating IgE responses in allergic individuals and the isolation and synthesis of immunoglobulin class-specific helper and suppressor factors, therapeutic intervention to recreate the homeostatic balance of oral food tolerance can be undertaken.

The author would like to thank the National Fund for Research into Crippling Diseases for generous grant support.

\section{REFERENCES}

Bienenstock, J. (1982). In Clinics in Immunology and Allergy, vol. 2, pp. 5-14 [J. Brostoff and S. J. Challacombe, editors]. London: W. B. Saunders.

Bloch, K. J. \& Walker, W. A. (1981). Journal of Clinical Immunology 67, 312-316.

Chalon, M. P., Milne, R. W. \& Vaerman, J. P. (1979). European Fournal of Immunology 9, $747-75^{1}$.

Cunningham-Rundles, C., Brandeis, W. E., Good, R. A. \& Day, N. K. (1978). Proceedings of the National Academy of Sciences 75, 3387-3380.

Egger, J., Carter, C., Wilson, J., Turner, M. W. \& Soothill, J. F. (r 984). Lancet ii, 865-869.

Jarrett, E. E. E., Haig, D. M., McDougall, W. \& McNutty, E. (1976). Immunology 30, 671-677.

Owen, R. L. \& Jones, A. I. (1974). Gastroenterology 66, 189-203.

Paganelli, R., Atherton, D. A. \& Levinsky, R. J. (1983). Archives of Diseases in Childhood $\mathbf{5}^{8}$, $20 \mathrm{I}-206$.

Paganelli, R. \& Levinsky, R. J. (1981). Scandinavian fournal of Immunology 14, 353-358.

Richman, L. K., Graeff, A. S., Yarchoan, R. \& Strober, W. (1981). Fournal of Immunology 126, 2079-2083.

Roberton, D., Paganelli, R., Dinwiddie, R. \& Levinsky, R. J. (1982). Archives of Diseases in Childhood $57,3^{69-372 .}$

Russell, M. W., Brown, T. A. \& Mestecky, J. (1981). Journal of Experimental Medicine 153, $968-976$.

Strobel, S., Mowat, A. M., Drummond, H. E., Pickering, M. G. \& Ferguson, A. (1983). Immunology 49, 451-456.

Tryphonas, H. \& Trites, R. (1979). Annals of Allergy 42, 22-26.

Walker, W. A. (1982). In Clinics in Immunology and Allergy, vol. 2, pp. 15-40 [J. Brostoff and S. J. Challacombe, editors]. London: W. B. Saunders. 